\title{
El Mercado de Valores como fuente de financiamiento para LAS PEQUEÑAS Y MEDIANAS EMPRESAS ECUATORIANAS
}

\author{
The Stock Market as a source of FinANCING FOR SMALl AND \\ MEDIUM SIZED ECUADORIAN ENTERPRISES
}

PhD. Jesús F González Alonso

Universidad Tecnológica Israel jesusgalonso@uisrael.edu.ec

Ing. Juan Pablo Nieto Aguas Correo electrónico: juanpablo3639@hotmail.com

\section{Resumen}

La búsqueda de recursos, principalmente económicos, que permita el crecimiento de de los sectores industrial, empresarial y comercial dio lugar a la creación de dos grandes mercados. El uno, el Mercado Financiero, conformado por instituciones que ofertan préstamos de dinero a cambio de un porcentaje de interés. Y el segundo, el Mercado de Valores en donde la empresa obtiene financiamiento directamente de sus inversionistas. 
En el Ecuador, oficialmente, desde la creación de las Bolsas De Valores existe la posibilidad de que las pequeñas y medianas empresas obtengan recursos financieros valiéndose del Mercado de Valores. La poca divulgación e información existente y también en gran parte por temor a lo complejo, miedo al cambio y apego a lo tradicional, no ha permitido a las pequeñas y medianas empresas aprovechar todos los beneficios que les puede brindar el mercado bursátil.

Durante el desarrollo del trabajo de investigación, se ha determinado que la posibilidad que tienen las pequeñas y medianas empresas de obtener recursos financieros valiéndose del mercado de valores, es real, existe y es factible para las organizaciones legalmente constituidas.

Palabras claves: Financiamiento, Mercado de valores, Pequeña Empresa, Mediana Empresa, Ecuador.

\begin{abstract}
:
The search for resources, mainly economic, that allows the growth of the industrial, business and commercial sectors led to the creation of two large markets. The one made by the Financial Market lending institutions offering money in exchange for a percentage of interest. And the second, the stock market where the company obtains funding directly from its investors.

In Ecuador, officially, since the establishment of stock exchanges there is a possibility that small and medium-sized enterprises taking advantage obtain financial resources Securities Market. The little existing information dissemination and also largely for fear of the complex, fear of change and attachment to traditional, has prevented small and medium enterprises to leverage all the benefits that can provide them the stock market.

During the development of the research work it has been determined that the possibility for small and medium enterprises to obtain financial resources making use of the stock market is real, exists and is feasible for legally constituted organizations.
\end{abstract}

Keywords: Financing, Stock Market, Small company, Medium-sized enterprise, Ecuador. 


\section{Resultados}

\section{Conociendo el mercado de valores}

Para empezar a entender de una mejor manera el funcionamiento y la razón de ser que tienen los mercados de valores o bursátiles, se considera importante describir de los siguientes elementos:

- Sistema financieros.

- Mercado financiero.

- Instituciones financieras.

- Mercado de valores.

- Organismos de control.

- Integrantes del Mercado de Valores.

\section{Sistema Financiero}

Está formado por el conjunto de instituciones, medio y mercado, cuyo fin primordial es canalizar el ahorro que generan las unidades de gasto con superávit, hacia los prestatarios o unidades de gasto con déficit. El sistema financiero comprende, pues, tanto los instrumentos financieros o activos financieros, como las instituciones o intermediarios y los mercados financieros: los intermediarios compran y venden los activos en los mercados financieros (Calvo, 2010, p.35).

Entonces el sistema financiero es un conjunto de métodos y actividades orientadas a la compra y venta de activos o instrumentos financieros. Además contempla elementos de control que, entre otras cosas, ayudan a fijar un precio justo. Se constituye en el mecanismo por medio del cual se canaliza los recursos monetarios, así como los excedentes financieros hacia actividades económicas y productivas.

Según la clasificación concerniente al tema de investigación. El sistema financiero se divide en:

- Instituciones Financieras.

- Mercado De Valores. 


\section{Instituciones Financieras}

Las instituciones financieras en nuestro país se encuentran formadas principalmente por el sistema bancario. Los bancos obtienen recursos por medio de la captación de dinero de sus clientes. Canalizan dichos recursos hacia, préstamos, inversiones y financiamiento de actividades económicas y productivas. Este concepto idealiza la razón de ser de un banco. Las instituciones bancarias se encuentran controladas por la superintendencia de bancos.

\section{Mercado Bursátil}

Un mercado de valores, o mercado financiero, se puede definir como un mecanismo que reúne a vendedores y compradores de activos financieros para facilitar las negociaciones. Una de sus principales funciones es la de descubrir el precio, es decir, garantizar que los precios de los valores reflejan la información disponible actualmente. Cuanto más rápido y con más precisión se alcance el precio del valor, los mercados financieros podrán dirigir con más eficiencia su capital hacia oportunidades más productivas, con lo cual se logrará un mejoramiento del bienestar público". (Gordon, 2010, p. 87).

\section{Mercado de valores}

El mercado de valores canaliza los recursos financieros hacia las actividades productivas, a través de la negociación de valores. Constituye una fuente directa de financiamiento y una interesante opción de rentabilidad para los inversionistas. (Bolsa de Valores de Quito, s.f).

\section{Ventajas del mercado de valores}

- Es un mercado organizado, integrado, eficaz y transparente; en el que la intermediación de valores es competitiva, ordenada, equitativa y continua, como resultado de una información veraz, completa y oportuna.

- Estimula la generación de ahorro, que deriva en inversión.

- Genera un flujo importante y permanente de recursos para el financiamiento en el mediano y largo plazo.

Se propone el concepto de que el mercado de valores es donde se negocian instrumentos financieros. Lo que dicho en otro contexto se entiende como la venta y compra de títulos valores, mismos que previamente fueron emitidos o colocados en el mercado por una empresa que necesita financiamiento. 


\section{Clasificación del mercado de valores}

Público: son las negociaciones que se realizan con la intermediación de una casa de valores autorizada.

Privado: son las negociaciones que se realizan en forma directa entre comprador y vendedor, sin la intervención de una casa de valores.

Primario: es aquel en el cual se realiza la primera venta o colocación de valores que hace el emisor con el fin de obtener directamente los recursos.

Secundario: comprende las negociaciones posteriores a la primera colocación de valores.

\section{Organismos de control}

El mercado de valores al ser un ente continuamente regulado por el sistema financiero presenta los siguientes organismos de control:

Consejo Nacional de Valores: es el órgano adscrito a la Superintendencia de Compañías que establece la política general del Mercado de Valores y regula su funcionamiento.

Superintendencia de Compañías: es la institución que ejecuta la política general del Mercado de Valores y controla a los participantes del mercado.

Bolsas de Valores: a través de su facultad de autorregulación pueden dictar sus reglamentos y demás normas internas de aplicación general para todos sus partícipes, así como, ejercer el control de sus miembros e imponer las sanciones dentro del ámbito de su competencia.

\section{Integrantes del Mercado de Valores}

El Mercado de Valores se encuentra conformado por elementos, participantes, actores, entidades, etc., que en conjunto hacen posible la existencia de un mercado bursátil.

Emisores: son compañías públicas, privadas o instituciones del sector público que financian sus actividades mediante la emisión y colocación de valores, a través del mercado de valores.

Inversionistas: son aquellas personas naturales o jurídicas que disponen de recursos económicos y los destinan a la compra de valores, con el objeto de lograr una rentabilidad adecuada en función 
del riesgo adquirido. Para participar en el mercado de valores no se requiere de montos mínimos de inversión.

Bolsas de Valores: son corporaciones civiles sin fines de lucro que tienen por objeto brindar los servicios y mecanismos necesarios para la negociación de valores en condiciones de equidad, transparencia, seguridad y precio justo.

Casas de Valores: son compañías anónimas autorizadas, miembros de las bolsas de valores cuya principal función es la intermediación de valores, además de asesorar en materia de inversiones, ayudar a estructurar emisiones y servir de agente colocador de las emisiones primarias.

Depósito Centralizado de Compensación y Liquidación de Valores: es una compañía anónima que se encarga de proveer servicios de depósito, custodia, conservación, liquidación y registro de transferencia de los valores. Opera también como cámara de compensación.

Calificadoras de riesgo: son sociedades anónimas o de responsabilidad limitada, independientes, que tienen por objeto la calificación de emisores y valores.

Administradoras de Fondos y Fideicomisos: son compañías anónimas que administran fondos de inversión y negocios fiduciarios. (Bolsa de Valores de Quito, s.f.).

Como se mencionó al comienzo de los resultados de la presente investigación, se trata de describir de una forma general los aspectos que conciernen al mercado de valores ecuatoriano, con el propósito de asimilar y entender su funcionamiento. Por esta razón es necesario en lo concerniente a los participantes, profundizar sobre las actividades y funciones de las bolsas y casas de valores.

\section{La Bolsa de Valores}

Las bolsas de valores son las herramientas vitales para el manejo de los mercados de capitales porque facilitan el financiamiento de diversos sectores económicos mediante el ahorro público, permitiendo la transferencia de recursos entre los que disponen de excedentes, hacia los que requieren de ellos para impulsar el desenvolvimiento de sus actividades, en el mediano y largo plazo. Las bolsas de valores constituyen el punto de encuentro donde compradores y vendedores negocian valores. (Bolsa de Valores de Quito, s.f.).

Se entiende que la Bolsa de Valores es el órgano de control del mercado bursátil. Haciendo referencia a las instituciones bancarias, funge como la Superintendencia de Bancos en relación. En el Ecuador existen dos bolsas de valores:

- La Bolsa de Valores de Quito.

- La Bolsa de Valores de Guayaquil. 


\section{La Bolsa de Valores en Ecuador}

En el Ecuador, son corporaciones civiles sin fines de lucro, autorizadas y controladas por la Superintendencia de Compañías, sujetas a las disposiciones de la Ley de Mercado de Valores y resoluciones expedidas por el Consejo Nacional de Valores.

Tiene por objeto brindar a sus miembros, las Casas de Valores, los servicios y mecanismos requeridos para la negociación de valores en condiciones de equidad, transparencia, seguridad y precio justo.

Tales operaciones se dan en el marco de un conjunto de normas y reglas uniformes y con el manejo de suficiente información.

\section{Funciones principales de la Bolsa de Valores de Quito}

- Proporcionar los mecanismos y sistemas que aseguren la negociación de valores de una manera transparente y competitiva.

- Mantener información actualizada sobre los valores cotizados, sus emisores, casas de valores y operaciones bursátiles.

- Controlar a las casas de valores miembros con el fin de dar cumplimiento a las normas reglamentarias y de autorregulación.

- Promover el desarrollo del mercado de valores.

\section{Autorregulación}

Es la facultad que tienen las bolsas de valores para dictar reglamentos y demás normas internas, así como para ejercer el control de sus miembros e imponer sanciones. La Bolsa de Valores de Quito expide sus regulaciones de carácter interno y operativo, las que requerirán únicamente de la aprobación del Directorio.

\section{Ventajas que proporciona la bolsa}

- Un menor costo de financiamiento.

- Un precio justo de compra y de venta.

- Liquidez permanente.

- Las condiciones de transparencia y la constante información en las negociaciones genera confianza.

- Información en línea de todas las transacciones que se realizan en los diferentes mecanismos de negociación.

- Diversificación del riesgo mediante las distintas alternativas de inversión y de financiamiento.

- Toma de decisiones adecuadas basadas en información veraz y oportuna. 


\section{Fondo de garantía de ejecución}

Es un fondo que se constituye con los aportes de las casas de valores y tiene por objeto asegurar el cumplimiento de las obligaciones de éstas frente a sus comitentes (clientes) y a la respectiva bolsa, derivadas exclusivamente de operaciones bursátiles.

Este fondo de garantía operará cuando las casas de valores:

- Utilicen indebidamente los valores o dinero entregados por los comitentes (clientes).

- Cuando ocasionen pérdidas a terceros por no cumplir con las normas de compensación y liquidación.

\section{Casas de Valores}

Son compañías anónimas autorizadas y controladas por la Superintendencia de Compañías, miembros de una Bolsa de Valores, cuyo objeto es la intermediación de valores. La intermediación de valores tiene por objeto vincular las ofertas y las demandas, para efectuar la compra o venta de valores. (Bolsa de Valores de Quito, s.f.).

Las casas de valor son miembros de las Bolsas de Valores. La principal características de éstas es que son las únicas autorizadas para realizar intermediación de valores; esto quiere decir que solamente por medio de éstas es posible negociar los diferentes títulos valores o instrumentos financieros que se ofertan en el mercado.

\section{Facultades de las casas de valores}

- Comprar y vender valores de acuerdo con las instrucciones de sus comitentes (clientes) en el mercado de valores.

- Adquirir o enajenar valores por cuenta propia.

- Dar asesoría e información en materia de intermediación, finanzas, estructuración de portafolios, adquisiciones, fusiones, escisiones y otras operaciones en el mercado de valores.

- Promover fuentes de financiamiento para personas naturales o jurídicas y entidades del sector público.

- Explotar su tecnología, servicios de información y procedimientos de datos y otros, relacionados con su actividad.

- Anticipar fondos de sus recursos a clientes, para ejecutar órdenes de compra.

- Efectuar actividades de estabilización de precios, únicamente durante la oferta pública primaria de valores.

- Realizar operaciones de Reporto bursátil.

\section{Prohibiciones de las casas de valores}

- Realizar actividades de intermediación financiera.

- Recibir por cualquier medio, captaciones del público. 
- Realizar actos o efectuar operaciones ficticias, o que tengan por objeto manipular o fijar artificialmente precios o cotizaciones.

- Garantizar rendimientos o asumir pérdidas de sus comitentes.

- Divulgar por cualquier medio, información falsa, tendenciosa, imprecisa o privilegiada.

\section{Responsabilidades de las casas de valores}

- La identidad y capacidad legal de sus comitentes.

- La existencia e integridad de los valores que negocien.

- La autenticidad del último endoso, cuando proceda.

- Realizar transacciones bursátiles a través de operadores inscritos en el registro de mercado de valores y en las bolsas de valores.

- Pagar el precio pactado de la compra o efectuar la entrega de los valores vendidos.

- Facilitar información a sus comitentes.

\section{Aspectos que usted debe tomar en cuenta respecto a las casas de valores}

- Son los únicos intermediarios autorizados para operar en Bolsa.

- Promover la movilización de pequeños y grandes capitales.

- Cobran una comisión por negociación de libre contratación.

- Son los únicos que pueden asesorar en materia de inversión, cuando ésta se realiza en la Bolsa.

A pesar de que Ecuador es un mercado pequeño que aún no ha logrado desarrollar una cultura bursátil entre empresas e individuos, existe un gran número de casas de valores, alrededor de 40 a nivel nacional. Sin duda una cifra exagerada tomando en cuenta el tamaño y potencial que tiene el mercado, por lo que también resulta difícil asimilar que existan dos bolsas de valores con reglamentos distintos.

A continuación, entre otras, se enumeran algunas casas de valores que operan en el mercado:

- Valorapolo casa de valores Apolo.

- Ecuabursatil casa de valores.

- Accival casa de valores.

- Analytica Securities casa de valores.

\section{Instrumentos Financieros}

Durante el desarrollo del trabajo se ha referido muchas veces al término negociaciones. Los instrumentos financieros o títulos valores precisamente son los elementos que se negocian en el mercado de valores. 
Los títulos valores precisamente constituyen la técnica que utilizan las empresas para obtener financiamiento en el mercado de valores. Esta figura toma el nombre de emisión de deuda.

Entonces la emisión de deuda se la puede definir como el mecanismo de colocar o emitir en el mercado títulos valores por parte de una empresa con el propósito de que estos sean adquiridos por inversionistas y de esta manera obtener financiamiento.

Se considera valor al derecho o conjunto de derechos de contenido esencialmente económico, negociables en el mercado de valores. Este derecho puede estar representado en títulos o en registros contables o anotaciones en cuenta.

Según el tipo de valor, éste puede representar un crédito a favor de su titular o del poseedor del mismo y por lo tanto significa una deuda a cargo de quien lo haya emitido. Puede también constituir un reconocimiento de participación patrimonial en la entidad emisora.

Para que un derecho de contenido económico sea considerado como un valor negociable, debe ser reconocido como tal por el Consejo Nacional de Valores. (Bolsa de Valores de Quito, s.f.).

\section{Tipos de instrumentos financieros}

Según la característica que determina la rentabilidad del título para el inversionista, en el mercado existen dos tipos de instrumentos financieros, estos son:

- Títulos de renta fija.

- Títulos de renta variable.

\section{Títulos de renta fija}

Son aquellos cuyo rendimiento no depende de los resultados de la compañía emisora, sino que está predeterminado en el momento de la emisión y es aceptado por las partes.

En este tipo de títulos se establece claramente el monto, el plazo y la tasa de interés que éste generará en el periodo estipulado de tiempo. La rentabilidad de este tipo de valores viene dada por:

- El valor de los intereses ganados o por ganar, en función de la tasa establecida en la emisión y el plazo correspondiente.

- El valor del descuento o premio que se obtenga en el precio de negociación en el mercado. 
El pago periódico de los intereses y de capital en algunos valores de renta fija, se hace mediante cupones, que son valores desprendibles emitidos de forma conjunta con los valores principales, reconocen dicho pago, en una fecha determinada y la suma de dinero que corresponde al monto de intereses devengados en un periodo establecido. Estos cupones pueden negociarse junto con el valor principal o en forma independiente, según la necesidad y conveniencia del tenedor.

Para otros valores que no tienen cupones, el pago se realiza al vencimiento del plazo, junto con el capital, se los denomina valores cero cupón.

Con el fin de extender más el estudio de las características que abordan los diferentes títulos valores, para la presente investigación, es necesario establecer los principales que se emiten y negocian en el Ecuador por parte de empresas privadas. Por tal motivo, referente al estudio de títulos de renta fija, se ha considerado profundizar el estudio de los siguientes instrumentos financieros:

- Obligaciones.

- Papel Comercial.

- Titularizaciones.

\section{Obligaciones}

Las obligaciones son valores que son emitidos como un mecanismo que permite captar recursos del público y financiar sus actividades productivas. De tal manera que las obligaciones son valores representativos de una deuda, que el emisor reconoce o crea, y que son exigibles según las condiciones de la emisión.

Como título de crédito de derecho al cobro de intereses y a la recaudación del capital, para lo cual podrán contener cupones. Se consideran obligaciones de largo plazo cuando el plazo de los valores es superior a trescientos sesenta días contados desde su emisión hasta su vencimiento. Las obligaciones de largo plazo son los valores que pueden ser emitidos por:

- Compañías anónimas.

- Compañías de responsabilidad limitada.

- Sucursales de compañías extranjeras domiciliadas en el Ecuador.

- Organismos seccionales que reconocen o crean una deuda a cargo de la emisora.

Se concluye que las obligaciones son títulos valores o instrumentos financieros considerados a largo plazo, mayores a un año. El pago es por medio de cupones de capital e interés. La empresa que los emite establece la cantidad de la emisión y los términos y condiciones del título, así como la forma y el lugar de pago. 


\section{Papel Comercial}

El papel comercial es un tipo de obligación pero de corto plazo, por cuanto deberán ser emitidos con plazos inferiores a 360 días. Las compañías emisoras inscritas en el Registro del Mercado de Valores podrán emitir obligaciones de corto plazo.

\section{Revolvencia del papel comercial}

La Superintendencia de Compañías conferirá a los emisores, previo acuerdo de junta general de accionistas o de socios, un cupo de emisión revolvente lo que implica que la empresa podrá redimir, pagar, recomprar, emitir y colocar papel comercial para que, de forma continua y de acuerdo a sus necesidades de fondos, mientras esté vigente esta autorización, emitan papel comercial en los términos de la Ley de Mercado de Valores, dentro del monto y plazo autorizados.

Se concluye que los papeles comerciales son títulos valores o instrumentos financieros consideradas obligaciones a corto plazo, menores a un año. Este tipo de título es muy parecido al producto conocido como póliza de acumulación que oferta el Sistema Bancario.

\section{Titularización}

Es un proceso jurídico que convierte las expectativas de flujo de fondos futuros en títulos negociables hoy. Todo buen proyecto futuro puede obtener recursos al momento actual.

Esta modalidad abre campo para que los más diversos tipos de activos ilíquidos puedan ser movilizados a través de la venta de los títulos que los representan. Emitidos con cargo a un patrimonio autónomo.

Los valores producto de este proceso son susceptibles de ser colocados y negociados libremente en el mercado bursátil.

\section{Tipos de titularización}

Existen 4 tipos de titularización, pero el más conocido y utilizado en el mercado ecuatoriano es aquel que se fundamenta como garantía la cartera de clientes que mantiene la empresa.

- Titularización de cartera.

- Titularización de inmuebles.

- Titularización de proyectos inmobiliarios.

- Titularización de flujos de fondos en general. 


\section{Titularización de cartera}

La titularización de cartera consiste en la venta a inversionistas de la cartera que han puesto los intermediarios financieros. Vender una cartera significa ceder los derechos al pago del principal y de los rendimientos a los inversionistas que la compra. El proceso de titularización de cartera solo se podrá estructurar con carteras de la misma clase; no se aceptarán mezclas ni combinaciones de carteras.

\section{Titularización de inmuebles}

La Titularización de inmuebles se perfecciona mediante un contrato de fiducia mercantil irrevocable de garantía o de administración, el originador transfiere a un patrimonio autónomo uno o varios bienes inmuebles de baja rotación, con cargo al cual la sociedad fiduciaria emite los títulos.

\section{Titularización de proyectos inmobiliarios}

La titularización de proyectos inmobiliarios consiste en la emisión de títulos mixtos o de participación que incorporen derechos alícuotas o porcentajes sobre un patrimonio de propósito exclusivo constituido con un bien inmueble, los diseños, estudios técnicos y de pre factibilidad económica, programación de obra y presupuestos necesarios para desarrollar un proyecto inmobiliario objeto de titularización.

El inversionista es partícipe del proyecto en su conjunto, obteniendo una rentabilidad derivada de la valoración del inmueble, de la enajenación de unidades de construcción o, en general, del beneficio obtenido en el desarrollo del proyecto.

\section{Titularización de flujos de fondos en general}

La titularización de flujos de fondos consiste en el traspaso al patrimonio autónomo del derecho de cobro de los flujos de efectivo previsibles en el futuro. Se podrán estructurar procesos de titularización a partir de la transferencia al patrimonio autónomo del derecho de cobro de flujos futuros de fondos determinables con base en información histórica de al menos los últimos tres años y en proyecciones de por lo menos tres años consecutivos, según corresponda, por el plazo y la vigencia de los valores a emitirse.

\section{Valores de Renta Variable}

Son el conjunto de activos financieros que no tienen un vencimiento fijo y cuyo rendimiento, en forma de dividendos y capital, variará según el desenvolvimiento del emisor. 
Tanto en el mercado primario como secundario, los valores de renta variable, inscritos en bolsa deberán negociarse únicamente en el mercado bursátil, a través de las casas de valores, con excepción de las transferencias de acciones originadas en fusiones, escisiones, herencias, legados, donaciones y liquidaciones de sociedades conyugales o de hecho.

Dentro del grupo de instrumentos financieros con rentabilidad variable se identifica los siguientes títulos:

- Acciones.

- Cuotas de participación.

\section{Acciones}

Habitualmente se les define a las acciones como la parte alícuota del capital social de la empresa que las ha emitido. Por tanto, poseer una acción de una empresa significa ser propietario de la misma en una porción igual a la que esa acción representa sobre el total de acciones emitidas”. (Brun, 2008, p.14).

\section{Rendimiento de las acciones}

El rendimiento de una acción depende del resultado del balance anual de una compañía, siendo repartible únicamente el beneficio líquido y efectivamente percibido. En consecuencia, las acciones no pueden garantizar un rendimiento fijo. El beneficio que proporcionan las acciones es variable, este beneficio que recibe el accionista se llama dividendo. Podrán emitir acciones:

- Las sociedades anónimas y de economía mixta que se funden mediante constitución sucesiva o suscripción pública de acciones.

- Las sociedades anónimas y de economía mixta existentes que realicen aumentos de capital por suscripción pública.

\section{Cuotas de Participación}

Representan los aportes realizados por los constituyentes de un fondo colectivo, y que son valores negociables en el mercado y requieren de una calificación de riesgo.

\section{Revni}

Es un mecanismo establecido y autorregulado por las Bolsas de Valores por el cual las empresas que no se encuentran inscritas en el Registro de Mercado de Valores ni en Bolsa, pueden anotar valores en un registro especial con la finalidad de cotizar sus valores de manera ocasional o periódica. 
Para obtener la autorización de anotación en el Registro de Valores no Inscritos (Revni), se deberá presentar una solicitud suscrita por el representante legal del emisor de los valores dirigido a la Presidencia Ejecutiva o a la Dirección General, según corresponda, para que inicie el trámite de anotación en la Bolsa de Valores, receptora de la solicitud. La solicitud de anotación deberá contar con el auspicio de un intermediario de valores.

Se concluye que el Revni (Registro Especial de Valores No Inscritos) es un caso particular, ya que la empresa que los emite no necesariamente debe estar registrada en la bolsa de valores. El revni puede adoptar las características de un título de renta fija, renta variable, a largo o corto plazo. Sin embargo la manera que más se lo utiliza en Ecuador es en forma de pagaré. Los pagarés son instrumentos financieros de renta fija. (Bolsa de Valores de Quito, s.f.).

Se ha concluido con la descripción y estudio de los principales conceptos, componentes y participantes que en conjunto forman el mercado de valores ecuatoriano. Este análisis previo ha sido necesario con el fin de entablar una idea más clara y amplia del funcionamiento del mercado. Con éste análisis ya resulta posible comprender de qué manera las empresas pueden beneficiarse de los distintos mecanismos y canales que ofrece el Mercado de Valores para obtener financiamiento. Ahora el objetivo de la investigación se orienta en identificar cuál de las distintas alternativas de financiamiento se constituye como la mejor opción para las pequeñas y medianas empresas.

\section{Las pequeñas y medianas empresas}

Se conoce como pymes al conjunto de pequeñas y medianas empresas que de acuerdo a su volumen de ventas, capital social, cantidad de trabajadores, y su nivel de producción o activos presentan características propias de este tipo de entidades económicas. Por lo general, en nuestro país las pequeñas y medianas empresas que se han formado realizan diferentes tipos de actividades económicas entre las que destacamos las siguientes:

- Comercio al por mayor y al por menor.

- Agricultura, silvicultura y pesca.

- Industrias manufactureras.

- Construcción.

- Transporte, almacenamiento, y comunicaciones.

- Bienes inmuebles y servicios prestados a las empresas.

- Servicios comunales, sociales y personales.

\section{Pequeñas empresas}

Las pequeñas empresas son aquella unidad productiva que tenga entre 10 a 49 trabajadores, un valor de ventas o ingresos brutos anuales entre cien mil y un millón de dólares americanos y/o un volumen de activos entre cien mil uno y setecientos cincuenta mil dólares. (Gobierno de Pichincha, s.f.). 


\section{Medianas empresas}

Aquella unidad productiva que tenga entre 50 a 159 trabajadores, un valor de ventas o ingresos brutos anuales entre un millón uno y cinco millones de dólares americanos y/o un volumen de activos entre setecientos cincuenta mil uno y cuatro millones de dólares. (Gobierno de Pichincha, s.f.).

\section{Importancia de las pymes}

Las pymes en nuestro país se encuentran en particular en la producción de bienes y servicios, siendo la base del desarrollo social del país tanto produciendo, demandando y comprando productos o añadiendo valor agregado, por lo que se constituyen en un actor fundamental en la generación de riqueza y empleo. (Servicios de Rentas Internas, s.f.).

Las pequeñas y medianas empresas dinamizan la economía nacional, puesto que son numerosas y diversas. Cuentan además con una amplia gama de actividades económicas y productivas dentro de las cuales se desenvuelven. Se constituyen en poderosas fuentes de empleo y colaboran notablemente al crecimiento económico del país. Las pequeñas y medianas empresas se encuentran limitadas al momento de conseguir fuentes de financiamiento y éstas requieren financiamiento constante ya que están encaminadas a una incesante expansión comercial debido a lo competitivo y cambiante que puede volverse el mercado en el que se desenvuelven.

La mayoría de estas organizaciones consideran la opción de un préstamo bancario como la única opción para obtener recursos financieros. Desconocen que existen otros mecanismos diferentes, como por ejemplo, el que ofrece el Mercado de Valores.

\section{Financiamiento por medio de la banca}

Es entonces necesario analizar las características que tiene un préstamo bancario otorgado a las pequeñas y medianas empresas. La investigación determinó que la tasa de interés activa; es decir el porcentaje de interés que cobra la institución bancaria, promedio para una pymes bordea el 12\% anual.

En referencia a los plazos en préstamos que otorga el sistema bancario, generalmente bordean entre 1 y 2 años. Estas condiciones, de interés y plazo, no resultan precisamente las más favorables para los empresarios, sin embargo la adoptan por desconocer la existencia de otras fuentes de financiamiento.

\section{Financiamiento por el Mercado de Valores}

El Mercado de Valores ofrece la posibilidad de obtener recursos financieros emitiendo deuda. La emisión de deuda se la hace por medio de instrumentos financieros colocados en la bolsa de valores. 
Dicho en un contexto más práctico, la emisión de deuda se da cuando una organización coloca o emite al mercado títulos valores (acciones, obligaciones, papel comercial, titularizaciones, Revni, etc.), con el propósito de ser negociados y adquiridos por inversionistas. Es por esta razón que dichas organizaciones toman el nombre de emisores.

Las empresas, al obtener financiamiento en el mercado de valores por medio de la emisión, al contrario de un préstamo bancario, son ellas las que deciden e imponen el monto, la tasa de interés y el plazo. Generalmente las tasas oscilan fluctúan entre los valores del $6 \%, 7 \%, 8 \%$ y $9 \%$. Se debe cuidar no fijar una tasa demasiado baja ya que no sería atractiva para el inversionista, lo que conllevaría a demoras al momento de conseguir financiamiento.

\section{Ventajas para el inversionista}

La tasa de interés pasiva promedio para inversiones en la banca bordea el $4 \%$ anual. Este porcentaje no resulta muy atractivo para las personas que desean sacar el máximo provecho a su dinero, por lo que los productos bancarios no consiguen satisfacer completamente sus necesidades.

Por otro lado, si un inversionista adquiere algún instrumento financiero, en el mercado de valores, la tasa de interés que genera su inversión bordearía el $8 \%$ de interés sobre el capital, el doble que en una institución bancaria y esta cifra puede subir considerablemente dependiendo del título valor adquirido.

\section{Método Win Win}

En técnicas de negociación se presenta el modelo Win - Win (ganar - ganar) cuando las dos partes resultan beneficiadas de la transacción y logran satisfacer sus necesidades.

El modelo Win -Win efectivamente se evidencia en el mercado de valores. La razón se debe a que la empresa en lugar de pagar el 12\% de interés por un préstamo bancario solo pagaría el $8 \%$ al inversionista sin olvidar que ésta es la que decide las condiciones de pago. Por otro lado, el inversionista en lugar de recibir el $4 \%$ por una inversión en una institución bancaria recibiría el 8\%. Al adquirir un título en el mercado de valores.

\section{Propuesta de financiamiento para Pymes}

Una vez que contamos con una idea clara y más amplia referente al mecanismo de emisión de deuda y la manera de obtener financiamiento valiéndose del mercado bursátil, es tiempo de determinar cuál de los diferentes instrumentos financieros (títulos valores) que existen, es el que mejor se ajusta a los requerimientos y necesidades de las pequeñas y medianas empresas. 
Conocemos que los instrumentos financieros más utilizado por las empresas en nuestro país son: acciones, obligaciones, titularizaciones, papel comercial y el Revni. También entendemos a que se refieren cada uno de ellos, sus características particulares así como requisitos, necesario cumplir por la empresa, para ser emitidos.

Después del análisis correspondiente se ha llegado a la conclusión que la opción más adecuada y conveniente de título valor o instrumento financiero, para que una Pyme coloque en el mercado, corresponde al revni en forma de pagare.

Los revnis corresponden a los registros especiales de valores no inscritos en bolsa. Este tipo de instrumento financiero es el que menos requisitos requiere para su emisión, en comparación a las otras opciones.

Es importante recordar que el revni tiene la particularidad, al contrario de los títulos valores analizados, que las empresas que lo emiten no necesariamente deben estar registradas en el Mercado de Valores, peculiaridad que ahorra de manera considerable costos, tiempo, trámites y requisitos.

Los revnis representan el primer acercamiento de una empresa al mundo bursátil, por lo que los requisitos solicitados no resultan tan complejos y complicados como los requeridos para emitir acciones, titularizaciones, obligaciones o papeles comerciales, éstos realmente ya se encuentra enfocados para empresas grandes, con gran capital social, con una trayectoria positiva en el mercado, sólidamente financieras y confiables. Estás características no se ajustas necesariamente a la realidad de una pequeña o mediana empresa.

La investigación develó que precisamente existe un producto o mejor dicho una iniciativa, amparada en el revni, llamado "Mipyme Bursátil" que motiva e incentiva a que este tipo de empresas ingresen y se beneficien del mercado de valores.

\section{MiPyme Bursátil}

Es un programa que ha sido lanzado y enfocado con el propósito específico de que las pequeñas y medianas empresas se beneficien del mercado de valores obteniendo financiamiento por medio de éste.

La falta de información y divulgación de este interesante mecanismo por parte de las autoridades correspondientes, una vez más, ha ocasionado el desconocimiento por parte de las empresas de esta acertada iniciativa. Un extracto de la información proporcionada por la bolsa de valores de Quito (BVQ) referente a MiPyme Bursátil se describa a continuación:

Al detectarse que el financiamiento para las pequeñas y medianas empresas es uno de los problemas más apremiantes para su desarrollo, es que la BVQ impulsó nuevas maneras de aprovechar las ventajas del mercado bursátil para empresas constituidas y de tamaño más pequeño así como los emprendimientos prontos a estrenarse. 
Conseguir recursos acordes al flujo propio de cada negocio es posible en el mercado de valores a través de ese mecanismo. Las bolsas de valores mantienen un registro especial en el que se anotan los valores no inscritos que se cotizan ocasionalmente en el programa "MiPyme Bursátil".

"Este mecanismo ha sido especialmente diseñado para pequeñas y medianas empresas que requieren financiamiento acorde a su realidad", explica Mónica Villagómez, Presidenta Ejecutiva de Bolsa de Valores de Quito (BVQ).

Así, las exigencias se adecuan a las características de las MiPymes sin descuidar procedimientos básicos que permitan incluir a jugadores serios en el mercado de valores. "Si bien no se pide a los actores que utilizan "MiPyme Bursátil" una calificación de riesgo, por el costo que ella implica, sí se solicita información legal y económica, auditada, pormenorizada y actualizada, que permita comprender el riesgo de los valores a anotarse", añade Villagomez.

También significa un ahorro de tiempo y dinero pues la inscripción se la hace directamente en la bolsa y no tiene costo. "MiPyme Bursátil" es un mecanismo más liviano, pero no por eso menos formal e informado. Con él se busca que las pequeñas y medianas empresas logren beneficiarse de las ventajas de participar en el mercado de valores: tasas más convenientes plazos más largos y condiciones de pagos establecidas por el cliente.

\section{Cómo anotar en MiPyme Bursátil}

El primer paso es trabajar junto a una casa de valores que auspicie la anotación y que asesore a la MiPyme sobre cómo organizar la información y estructuración de la anotación. Se anotan valores que no están inscritos en el Registro de Mercado de Valores. Estas anotaciones se encuentran respaldadas con garantía general, esto es el $80 \%$ de los activos libres de gravamen, es decir que no están prendados ni hipotecados.

La casa de valores es la encargada de ordenar la documentación y efectuar la estructuración legal y financiera para solicitar la anotación.

La documentación que la empresa debe presentar es bastante sencilla, sin embargo, demuestra al inversionista que la empresa cumple con sus obligaciones (pagos al IESS, impuestos, otros). Por otro lado, la empresa debe presentar estados financieros auditados cuando fuere aplicable, caso contrario deberá presentar los estados financieros del último año debida suscritos por el representante legal y su contador.

Las anotaciones tienen una vigencia de 180 días con la posibilidad de extenderse por 180 días más.

Pasos para aprovechar MiPyme Bursátil:

1. Buscar una casa de valores que asesore a la micro, pequeña, mediana empresa. 
2. Entregar información financiera y legal a la Casa de Valores para iniciar la estructuración legal y financiera.

3. Solicitar la anotación de los valores en el Registro Especial de Valores no Inscritos (Revni)

4. Publicar el "Extracto de solicitud aprobatoria" en un medio de amplia circulación.

5. Negociar los valores anotados y obtener financiamiento.

\section{Conclusiones}

La emisión de deuda por medio de instrumentos financieros constituye en la opción que ofrece el mercado de valores para que las empresas accedan a nuevas fuentes de financiamiento. Los registro de valores no inscritos (revni) son los más recomendables para acercase al mercado bursátil. Las ventajas de obtener financiamiento por emisión de deuda se ajustan a las necesidades de las empresas en términos de monto, tasa y plazo. La poca difusión de los beneficios, que ofrece ésta alternativa que brinda el sistema financiero, suscita en el desconocimiento de los empresarios e incluso la falta de interés por ser considerado por muchos un tema elitista.

Hoy en día, la mayoría de empresas parecen tener una solo opción de financiamiento. El tradicional préstamo que otorga las instituciones bancarias es el mecanismo más usado para obtener recursos. Es el más conocido y para muchos empresario el único sistema de financiamiento que existe. Esta idea sobre todo se presenta en las empresas pequeñas que se manejan desde un punto de vista familiar. En las grandes empresas si es posible evidenciar que existe conocimiento para aprovechar las ventajas que se puedo obtener a financiarse a través del mercado bursátil.

\section{Recomendaciones}

Diseñar y ejecutar por parte de las autoridades correspondientes un programa continuo de divulgación que aborde los beneficios que ofrece el mercado de valores ecuatoriano a empresas e inversionistas. Se considera importante también orientar la información con otro enfoque. Difundir desde la etapa escolar de un individuo servirá para que la sociedad se familiarice con este concepto y lograr una verdadera cultura bursátil en nuestro país al igual que posee las naciones consideradas de primer mundo.

Se recomienda a las pequeñas y medianas empresas utilizar diferentes alternativas de financiamiento. Los tradicionales préstamos bancarios no ofrecen los beneficios que brinda el mercado de valores. Es importante dinamizar la economía con un financiamiento que se ajusten mejor a las necesidades de las empresas. Es necesario aprovechar todas las oportunidades que ofrece el sistema financiero. 


\section{Bibliografía}

Berk, J., DeMarzo, P., Harford, J. (2010). Fundamentos de finanzas corporativas. Madrid: Pearson.

Block, S., Hirt, G., Danielsen, B. (2013). Fundamentos de administración financiera. México: McGraw Hill.

Bolsa de valores de Quito. (2010). Diccionario Búrsatil. Quito: Medium Multimedia.

Bolsa de valores de Quito. (2015). Conozca el mercado. Web oficial de la bolsa de valores de Quito. Fecha de consulta: 12 de Junio de 2015. URL: http://www.bolsadequito.info/inicio/conozca-el-mercado/

Bolsa de valores de Quito. (2015). Manuales de inscripción. Web oficial de la bolsa de valores de Quito. Fecha de consulta: 12 de junio de 2015. URL: http://www.bolsadequito.info/inicio/manuales-de-inscripcion-de-valores/

Brull, H. (2007). El mercado de capitales globalizado al alcance de todos. Argentina: Dunken.

Brun, X., Elvira, O., Puig, J. (2008). Mercado de renta variable y mercado de divisas. España: Bresca.

Calvo, A., Pareja, J., Rodriguez, L., Cueva, A. (2010). Manual del sistema financiero español. España: Ariel.

Cevallos, V. (1997). Mercado de valores y contratos. Quito: Edotorial jurídica del Ecuador.

Gitman, L. y Zutter, C. (2012). Principios de administración financiera. México: Pearson.

Gobierno de la provincia de Pichincha. (2013). Pymes agenda productiva provincial capítulo 4. Web oficial del gobierno de la provincia del Pichincha. Fecha de Consulta: 15 de junio del 2015. URL: http://www.pichincha.gob.ec/phocadownload/apoyoproduccion/ app_libro_4_pymesweb.pdf

Gordon, A. (2010). Fundamentos de inversión. México: Pearson.

Grinblatt, M. y Titman, S. (2010). Mercados financieros y estrategia empresarial. España: McGraw Hill.

Mankiw, G. (1999). Principios de economía. España: McGraw Hill.

Montalvo, M. (1998). Introducción al mundo de mercado de capitales. Quito: Imprima.

Ochoa, G. y Saldívar, R. (2012). Administración financiera correlacionada con las NIF. México: McGraw Hill.

Servicio de rentas internas del Ecuador. (2015). Pymes, Web oficial del Servicio de rentas internas. Fecha de Consulta 15 de junio de 2015. URL: http://www.sri.gob.ec/de/32

Superintendencia de Bancos. (2015). Tasa activa nominales promedio. Web oficial de la Superintendencia de Bancos. Fecha de consulta: 14 de junuio 2015, URL: http://www.sbs.gob.ec/practg/sbs_index?vp_art_id=153\&vp_tip=2 
